\title{
Nutraceuticals for Mangement of Cancer
}

\author{
Muhammad Imran Qadir $^{{ }^{*}}$, Saba Irshad $^{2}$ \\ ${ }^{1}$ Institute of Molecular Biology and Biotechnology, Bahauddin Zakariya University, Multan, Pakistan \\ ${ }^{2}$ Faculty of Pharmaceutical Sciences, Government College University, Faisalabad, Pakistan \\ *Corresponding Author: Muhammad Imran Qadir, Institute of Molecular Biology and Biotechnology, \\ Bahauddin Zakariya University, Multan, Pakistan, Tel: +92-301-6515613; Fax: +92-061-9210098; E-mail: \\ mrimranqadir@hotmail.com
}

Received: 12 May 2018; Accepted: 21 May 2018; Published: 06 June 2018

\begin{abstract}
It has been observed that $30-40 \%$ of the cancer can be avoided by only changing the life style and way of taking diet. Gerson Therapy advices a meal which is rich in salad, cooked vegetables, and potatoes which are highly baked soup and juices of vegetables. Increased intake of fruits reduce gastric cancer incidence.
\end{abstract}

Keywords: Nutritional advice; Vitamin A; Vitamin D

\section{Nutritional Advice for Treatment of Cancer}

It has been observed that $30-40 \%$ of the cancer can be avoided by only changing the life style and way of taking diet [1]. The best nutritional advice for treatment of cancer is:

1. Gerson Therapy; and

2. Treatment of cancer provided in Pennsylvania by the organization for advancement in cancer education.

\section{Gerson Therapy}

Gerson Therapy refers to the name of therapy that was presented by Max Gerson, who was a German physician. Gerson Therapy is regarded as an alternative therapy that has ability to cure cancer by the use of healthy diet.

\subsection{The advice of diet in gerson therapy}

Gerson therapy advices a diet which is rich in vitamins, micronutrients, enzymes, minerals and fluids, but consist of trace amount of fats. A typical routine diet according to Gerson therapy is given below. 


\subsubsection{Juices and Cancer:}

$>$ Carrot juice: Carrot juice contain vitamin A. Vitamin A deficiency increases the risk of cancer [2, 3].

$>$ Apple Juice: Apple Juice also contains vitamin A and C that decrease the risk of cancer [2, 3].

$>$ Juices obtained from green-leaf fresh organic fruits and vegetables: Those juices which are obtained from green-leaf fresh organic fruits and vegetables are very beneficial to heal cancer. Because these juices contains cellulose and carotenoides. Carotenoides helps in detoxification thus help in prevention of cancer [4].

2.1.2 Meal: Gerson Therapy advices a meal which is rich in salad, cooked vegetables, and potatoes which are highly baked soup and juices of vegetables. Increased intake of fruits reduce gastric cancer incidence [5]. Hence to heal cancer there must be availability of fresh fruits and fresh deserts fruit deserts for snacking in addition to regular diet [6]. Actually most of fruits and vegetables contain important anticarcinogens. Most of chemists have extracted more than 100 such chemical species from the plants [7].

2.1.3 Medication: Medications which are administered in Gerson therapy are all biological compounds that are obtained from organic compounds. Example of these compounds includes compounds of potassium, Vitamin B-12, Lugol's solution. Pancreatic enzymes, coffee enemas. Vitamin B-6 intake is inversely associated with risk of prostate cancer [6].

2.1.4 Enemas of coffee: Coffee stimulates the liver and helps to remove toxins in the body. Some practitioners recommend coffee enemas for detoxification $[8,9]$.

2.1.5 Detoxification: A remarkable alteration in food can produce seriously huge amounts of waste of cells in the stream of blood. That's why it's very important for a person to detoxify his system before and after the initial stages of diet. A person can detoxify his system by the use of castor oil which either act as additional stimulant of bile flow or increase the liver's ability to filter the blood. Digestive enzymes also help in detoxification by supporting the removal of damage tissue.

2.1.6 Use of water: Gerson therapy suggests that there should not be excessive use of water, because according to Gerson the stomach should be empty to allow digestion of juices and soups. Water usually contains trace quantities of the four trihalogenated methanes (THMs) chloroform, bromoform, bromodichloromethane, and dibromochloromethane. These compounds are considered to be responsible for cancer [10].

\section{Treatment of Cancer Provided in Pennsylvania by the Organization for Advancement in Cancer} Education

The center for advancement in cancer education in Pennsylvania is the one of the best center that provides important information about the treatment of cancer with the help of natural diet. 
Fortune J Health Sci 2018; 1 (1): 007-018

DOI: 10.26502/fjhs002

\subsection{General rule, of a diet to fight cancer}

The treatment of cancer provided in the Pennsylvania by the organization for advancement in cancer education suggests simply: high fiber, low animal protein, Diet that is rich in enzyme and unprocessed food has acid/alkaline ratio of 1:4, minimally processed organic food.

\subsubsection{Consumption of oils and fats}

Fatty food should be avoided. Food rich in fat increases the threat of different types of cancer, mainly the breast, colon, and to some extent the prostate cancer [11]. Diet that is rich in fats and cholesterol increases the risk of lung cancer [12]. Nevertheless, it has also been observed that Mediterranean diet is rich in fat and chances of breast cancer are very low there. Hence we can say that it is not only the fats that are important, but also the kind of fat plays a very important rule.

3.1.2 Animal's condition who's fat is being consumed: Animal's condition whose fat is being consumed should also be keep under consideration. Butter obtained from a traditionally raised cow is not better than organically raised cow. Monounsaturated fats mostly found (in sesame and olive seed) consumption should be high. But these must be used in unrefined form.

3.1.3 The consumption of oils after heat: The consumption of oils after heating must be avoided. The reason is that heat initiates the oxidation reactions with in the oil. These reactions cause the release of a large amount of free radicals. These free radicals increase the risk of cancer greatly. Researches made by The United State Department of Agriculture (USDA) suggested strongly that oil obtained from the fish decrease the Prostaglandin E2 production that has an effect of decreasing appetite. This decrease in appetite leads to condition called as scachexia, this syndrome during cancer ultimately leads to the death of patient.

\subsection{Elimination of cancer causing agents}

Any agent responsible for cancer should be eliminated. Following substances should be eliminated to treat cancer.

3.2.1 Consumption of Highly Processed food and risk of cancer: The substances produced due to over cook or processed foods are, polycyclic aromatic hydrocarbon, Heterocyclic amines, Dicarbonyls, Aminocarbonyls, is responsible for cancer [13].

3.2.2 Consumption of meat associated with risk of cancer: Increased intake of highly processed and red meat may increase the risk of colorectal cancer. Also it has been suggested that too much intake of seafood, white meat and dairy products should be avoided. 
Fortune J Health Sci 2018; 1 (1): 007-018

DOI: $10.26502 / f j h s 002$

3.2.3 White rice: The Japanese food mainly consists of increased intake of salty food as well as rice. However, it has been observed that the diet of Japanese people is being changing to Western diet, has resulted in increase risk of stomach cancer [14].

3.2.4 Consumption of sugar and risk of cancer: Carlo La Vecchia (1993) investigated that people who use sugar show an increased risk of colorectal cancer. Clear estimations has showed that People using one spoonful sugar has a risk of colon cancer 1.4, and those who use 2 spoonful sugar has a risk of developing cancer 1.6, and this risk is 2 for those people who use 3 spoonful sugar or more [17].

The cancerous cells require glucose (provided by sugar) as an essential component for survive. That's why most of tumour cells show an increased uptake of glucose (phenomenon known as the Warburg effect) [15, 16]. But their rates of oxidative phosphorylation are too much low. This causes the tumour cells to produce in the presence of oxygen, an increased concentration of lactate by the aerobic glycolysis [18]. That's why consumption of glucose should be avoided to cure cancer. The tumor cells produce lactic acid as an additional product. As a result instead of breaking the sugar to carbon dioxide and water tumor cells obtain their energy by converting the glucose to lactate. Accumulation of lactic acid in the tumour cells creates the tumour acidity [19].

The enzyme named as M2-PK has ability to convert the normal cells into cancerous cells [20]. M2-PK indicates the name of pyruvate kinase isoenzyme type M2. It exists in two forms the dimeric form as well as in the tetrameric form. However, the dimeric type of M2-PK predominates in cancerous cells, and causes the increased concentration of nucleic acid, amino acid and phospholipids, these compounds has an increased tendency of tumour growth [21]. This condition can be prevented by the use of a special class of sugar controlling molecules recognized as “Thiazolidinediones,” This class of sugar consist of anti-diabetic drugs called as Rosiglitazone and pioglitazone, that has a powerful ability to stop the production of PK-M2 enzyme.

3.2.5 Consumption of prickled or smoked foods: It has been revealed that the chances of cancer are greatly increased by the repeated intake of smoked or prickled food. These types of food mainly consist of polycyclic aromatic hydrocarbons and Nitrosamines. These compounds have a strong ability to create the risk of stomach and esophageal cancer. Among the smokers the increased consumption of alcohol causes an increased risk of cancer of esophagus, oral cavity, Respiratory tract and larynx [2].

3.2.6 Smoking and cancer: Tobacco smoke mainly consist of cancer causing agents such as, carbon monoxide, Ammonia, Tar, Sulfuric acid, Arsenic, Acetone, lead and Nicotine. More than 4,000 chemical compounds are known to present in tobacco smoke, out of which 43 chemical compounds have been extracted are responsible for development of cancer. Smoking has a strong role in development of cancer of mouth, esophagus, larynx, and lung, uterus, kidney, cervix, pancreas, bladder and stomach. 


\subsection{Food that we can use}

We can use fruits (dried and fresh but devoid of sulphurated compounds), white potatoes, vegetable juices, beans, raw vegetables, sweet potatoes, yogurt, and a minute amount of organically grown meat, poach fish, milks, nuts.

3.3.1 Cruciferous vegetable: A cruciferous vegetable is a special class of vegetables that shows excellent cancer fighting property [11]. The cancer fighting ability of cruciferous vegetables is mainly due to presence of phytochemical compounds, such as, isothiocyanates that causes the body to break down the impending carcinogenic cells as well as causes prevent the conversion of normal cells into tumour cells. Cruciferous vegetables also contain a compound called as, Indole-3-carbinol. Indole from this compound decreases the risk of breast cancer by either preventing an over production of a type of estrogen responsible for developing cancer or by promoting the conversion of type of estrogen responsible for cancer to a less harmful type. Another type of phytochemical obtained from cruciferous vegetables also has a special ability of neutralization of cancer causing agents before they can start tumour production. Cruciferous vegetables include vegetables like Bok choy, cabbage, Arugula, Broccoli, Chinese cabbage, Brussels sprouts, Cauliflower, Daikon, Kohlrabi, Kale, Collard greens, Mustard green, Turnips, Radishes, Watercress, and Rutabaga [22, 23].

3.3.2 Bioactive compounds of cruciferous vegetable: Bioactive compounds are obtained from cruciferous vegetables, such as Benzyl isothiocyanate and sulforaphane, Benzyl isothiocyanate and sulforaphane both are capable of inhibiting cell capability and inducing apoptosis (Programmed cell death is called apoptosis). Thus have inhibitory activity in pancreatic cancer cells [24].

3.3.3 Consumption of Broccoli and risk of cancer:Broccoli is considered to have a greatest cancer resisting ability than the other cruciferous vegetables, because of presence of a specific anticancer compound called as sulforaphane. Broccoli act as anticancer agent by switching ON the gene that prevent cancer development and switching OFF the other genes that initiate the cancer development.

3.3.4 Raw food: An uncooked and unprocessed plant food is known as Raw Food Diet. The raw food diet is an unprocessed and uncooked plant food. Such as, fresh food and vegetable, nuts, dried fruit etc. According to the researches of cancer experts, $70 \%$ of our total diet should be raw food diet [13]. The raw vegetables supply the important enzymes. However, no cancer expert suggests $100 \%$ of raw diet; the reason is that some vegetables also contain hard fibrous walls that can not be digested properly by our body if not properly broken down. We can break down the tough fibers of raw food without processing them by either shredding them or by running them through a food processor. But here one point should be kept in mind that is; food must be ingested immediately as it is processed through a processor, because it starts degenerating rapidly after processing.

\subsection{Components of cancer preventive diet}

Cancer experts recommended 50\% chemical elements in tumour avoiding diet consist of folic acid, Vitamin D, chlorophyll, Vitamin B-12, selenium 20, and antioxidants like carotenoids (lycopene, alpha-carotene, crytoxanthin, 
Fortune J Health Sci 2018; 1 (1): 007-018

DOI: $10.26502 / f j h s 002$

beta-carotene, lutein), Ascorbic acid [25]. However, Ascorbic acid shows partial benefits when administered orally, but it could be very beneficial when administered through intravenous injection [23]. Intake of digestive enzymes through oral cavity has also shown an important role to prevent cancer [23].

3.4.1 Beta-carotene: It has been observed that risk of developing lung cancer is considerably increased due to decrease beta-carotene blood serum level or blood plasma level. It means that beta-carotene is protective [4].

3.4.2 Vitamins A and C3: Vitamins A and C has also role in cancer prevention. Vitamin A deficiency enhances tumorigenesis.

\subsection{Purchase and preparation of food}

We should always try to purchase crisp, fresh and organic vegetables and should stay away from wilted things. Vegetables should be consumed immediately after their preparation. Leftovers should not be put in the refrigerators, because immediately after the cutting of vegetables, the enzymes and vitamins start to degenerate. Hence, only prepare those things which you can eat and throw out all the leftovers. To heal cancer we should avoid use of microwave oven and pressure cooker and should use glass, ceramic or steel cooking pots.

\subsection{Way of eating}

Never eat too much. Eat only when you are feeling hungry. Be seated and then eat consciously and slowly by chewing carefully and completely. During eating avoid talking.

\subsection{Tea intake and cancer risk}

Throughout this world tea is the commonly used beverage. Tea is obtained from a plant named as Camellia sinensis (this I is the specie of plant whose leaves and buds of leaves are employed for the production of tea). The tea obtained from camellia sinensis can be classified into black tea, green tea and oolong tea.

3.7.1 Green tea consumption and risk of cancer: The best intake of green tea is 3 - 5 cups/day. This is equal to round about $1200 \mathrm{ml}$ per day. By drinking 3-5 cups of green tea in a day becomes able to get $250 \mathrm{mg}$ per day of catechins (a chemical compound that is considers to be a powerful antioxidant). This concentration of catechins is considered to be safe but we should not exceed this limit [26]. It has been observed that consumption of green tea with in limits decreases the risk of prostate cancer. Drinking of green tea 2 cups in a day has the ability to decrease the risk of developing lung cancer up to $18 \%$. In the same way, the consumption of green tea also reduces the risk of rectum, esophageal, pancreatic, colon, liver, and gastric cancer.

Exceptions: It has been observed that green tea can increase the chances of cancer of urinary bladder in a person. 
3.7.2 How green Tea can reduce the risk of cancer?: Green tea is obtained from a plant known as Camellia sinensis (this is the specie of plant whose leaves and buds of leaves are utilized for the production of tea). Camellia sinensis consist of some active factors known as, polyphenol. The factor polyphenol is divided into a subclass called as, catechins. Catechins are considered to be strong antioxidants. Hence, the polyphenol of green tea has ability to eliminate the over growth of cancerous cells. Risk of prostate cancer can be decrease by the use of anti-oxidant polyphenolic compounds of green tea [27-29].

3.7.3 Black Tea and risk of Cancer: According to meta-analysis (In statistics, meta analysis can be defined as, a group of results of several studies that deals with a collection of research hypothesizes closely related to each other), black tea, cannot decrease the risk of lung cancer [30].

3.8 Intake of coffee and cancer risk

Drinking 2 cups of coffee in a day has the ability to enhance the chances of lung cancer up to $14 \%$. However, the risk of lung cancer can be decrease by consumption of decaffeinated coffee [8]. Coffee decreases the gastric cancer risk [31,32] the consumption of caffeinated coffee enhances the chances of colon as well as lung cancer mostly in males.

\subsection{Carbohydrates and risk of cancer}

It has been observed that food containing starch and oligosaccharides (present in sugar) enhances the chances of rectal cancer as well as colon cancer [33].

\subsection{Vegetables}

It has been proved that most of compounds of plant have the ability to prevent cancer. People that greatly depend upon fruits and vegetables have a reduced risk for the major cancers [34]. Vegetables intake can reduce prostate cancer [35]. Decrease consumption of carotenoides, vegetables and fruits has elevated risk of increase lung cancer [4]. $50 \%$ of total diet must be vegetables and vegetable juices. Carrot juice is the best cancer resisting juice. It is rich in alpha-carotene and beta-carotene, (However, alpha-carotene is ten times more strong as anti-oxidant agent than the beta-carotene). Vitamin A and C. Vitamin A and C and carotene have a special role in preventing cancer [2, 4, 36]. It has been found that Retinol (vitamin A) has ability to decrease the risk of larynx, bladder, mouth, pharynx, esophagus, rectum, stomach, colon cervix cancer [4]. Almost all the vegetables in the raw form can reduce the risk of rectal and colon cancer [33].

\subsubsection{Tomatoes and risk of cancer}

Tomatoes consist of a compound called as lycopene. This lycopene can decrease the chances of cancer [10]. Consumption of tomatoes can decrease the risk of cancer up to 30-40\% because of presence of lycopene. The risk of prostate cancer can be reduced up to $35 \%$ by the consumption of $2-4$ servings of tomato sauces in a week [9]. 
Fortune J Health Sci 2018; 1 (1): 007-018

DOI: 10.26502/fjhs002

3.10.2 Garlic consumption and risk of cancer

Several studies have shown that when the consumption of garlic is enhanced then the risk of several types of cancer is decreased like cancer of colon, breast, esophagus, stomach, and pancrease [37]. Cooked or raw garlic large quantity consumption may also decrease the chances of colorectal cancer [37]. The researches made by EPIC (European Prospective Investigation into Cancer and Nutrition has revealed that consumption of garlic and onion can greatly decrease the chances of Intestinal Cancer [38].

\subsubsection{How Garlic prevent from Cancer?:}

Garlic contains arginine, oligosaccharides, flavonoids, and selenium, these components helps to fight cancer. ${ }^{\text {[39], [40] }}$ it has been observed that selenium has a special role in eliminating the cancer.

Allyl Sulphur components of Garlic: Allyl Sulphur compounds of garlic can effectively inhibit the risk of cancer [41]. These components of garlic actually stop the nitrosamine formation and as a result reduction in carcinogen bioactivation occurs. Thus helps in preventing cancer [41, 42]. Allyl Sulphur components of garlic are soluble in lipids as well as water. These chemicals stop the tumor production by blocking a large quantity of chemical induced tumor. These chemicals can prevent the cancer of colon and skin. There is extensive evidence that components of garlic including diallyl sulfides can inhibit the induction of cancer in animals [43].

Flavonoids components of Garlic: Flavonoids components of garlic exhibit strong activities of killing cancerous cells. This property is exhibited due to presence of hydrogen donating free radical.

Fruits intake and risk of cancer: Our total diet should consist of $10 \%$ fruits. But raw fruits must be preferred. Citrus fruits consumption should be very low, because they consist of lactic acid. Lactic acid is responsible for increased chances of developing cancer [19]. Citrus fruit consumption must also be stopped because they consist of thioacetamide and thiourea. Thioasctamide and thiourea are the derivatives of hydrogen sulfide. These compounds are used to preserve the citrus fruits. These compounds cause the development of liver cancer and cancer of gall bladder [19].

\subsection{Animal Products}

The centre for advancement in cancer education advices to stop the consumption of red meat. And this centre prefers the white meat over red meat, like white meat of fish (particularly, salmon, cod, trout and haddock). It also restricts strictly the intake of that meat which is grown with the help of hormones and antibiotics. However, as red meat contains a large amount of iron, that have ability to react with the $\mathrm{O}_{2}$ to produce free radicals, which have anticancer effects. Hence, we can say that trace quantities of red meat can be used in soups, along with important antioxidants, like, Vitamin E and vitamin C. 
Fortune J Health Sci 2018; 1 (1): 007-018

DOI: 10.26502/fjhs002

\subsection{Consumption of Nuts and risk of cancer}

We must try to consume $5 \%$ of nuts and seeds of total diet. However, the nuts and seeds must be consumed in raw form. But those seeds and nuts that can put a strain on the digestive system must not be consumed in raw form but they should be consumed in somewhat processed form.

\subsection{Risk of cancer with the consumption of peanuts}

Peanuts are not nuts, but legume, these legumes may contain a common type of mold on them. These molds consist of carcinogenic strains that can cause the cancer. Hence, we can say that peanuts must be avoided in cancer.

\subsection{Soups}

Soups are a best source of breaking down the fibers in vegetables, and hence provide best nutritional components to treat cancer.

\subsection{Alkaline/acid balance}

According to Gerson any disturbance in acid/alkaline balance will leads towards an increase risk of cancer. Normally microorganisms, like, algae, nitrates, bacteria, heavy metals and bacteria causes the water to acidify. Acid environment of body initiates catabolic reactions in body, where as, Alkalinity leads towards anabolism. Alkaline environment of the body must be maintained for good health.

\subsection{Consumption of Fiber and Risk of Cancer}

A good diet to fight cancer must be high in fibers and low in fats. Fibers have ability to clean the Colon which is a part of large intestine. Hence, fibers decrease the chances of colon cancer. Usually in case of cancer of breast, two types of estrogen accumulate in body. The first one is bad estrogen and the second one is bad estrogen. Consumption of diet rich in fibers, have the ability to Decrease the bad level of Estradiol, hence, bad Estrogen helps to decrease the risk of cancer. It has been investigated that Consumption of fibers highly decreases the chances of Rectal Cancer.

\subsection{Risk of cancer with consumption of carotenoids}

Carotenoids are of several types. e.g. Alpha carotene and Beta carotene. Beta carotene is a strong anti cancer carotenoid. Beta carotene can be obtained from yellow vegetables, like, pumpkins, carrots and yams. Beta carotene was used in Russia as an effective agent to fight Leukemia in infant. Alpha carotene has ten times more anti cancer properties than Beta carotene. Most common source of carotenoids is Spinach.

\section{Conclusion}

It's much better to take dietary measures to prevent from the curse of cancer. Healing cancer naturally with food, nutrition and diet is beneficial. We should avoid all those things which are proved to be causing cancer and should take a balanced diet, for a healthy and peaceful life. 


\section{References}

1. Donaldson MS. Nutrition and cancer: a review of the evidence for an anti-cancer diet. Nutr J 3 (2004): 19.

2. Walker WN. Fresh Vegetables and Fruit Juices 30 Palmer S. Diet, nutrition, and cancer. Prog Food Nutr Sci 9 (2012): 283-341.

3. Zeigler RG. Vegetables, Fruits, and Carotenoids and risk of Cancer. AM J Clin Nutr 53 (1991): 251S-259S.

4. Lunet N, Lacerdass VA, Barros H. Fruit and vegetables consumption and gastric cancer: a systematic review and meta-analysis of cohort studies 53 (2005): 1-10.

5. Julie LK, Katja F, Lorelei AM, et al. One-carbon metabolism-related nutrients and prostate cancer survival. Am J Clin Nutr 90 (2009): 561-569.

6. Wargovich JM. New dietary anticarcinogens and prevention of gastrointestinal cancer. Disease of colon rectum 31 (1988): 72-75.

7. Tang N, WU Y, MA J, et al. Coffee consumption and risk of lung cancer: a meta-analysis 67 (2010): 17-22.

8. Botelho F, Lunet N, Barros H. Coffee and gastric cancer: systematic review and meta-analysis. Epub 22 (5): 889-900.

9. Crump KS, Guess HA. Drinking Water and Cancer 3 (1982): 339-357.

10. Palmer S. Diet, nutrition, and cancer. Prog Food Nutr Sci 9 (1985): 283-341.

11. Ziegler RG, Mayne ST, Swanson CA. Nutrition and lung cancer. Cancer causes control 7 (1996): 157-177.

12. McGrego D. Diet, food components and Human cancer. International agency for research on cancer 33 (2014): 189-196.

13. Osio T. Incidence of stomach cancer and its relation to dietary habits and nutrition in Japan between 1900 and 1975. Cancer res 35 (1975): 3254-3258.

14. Gogvadze V, Orrenius S, Zhivotoysky B. Mitochondria as targets for cancer chemotherapy. Semin Cancer Biol 19 (2008): 57-66.

15. Emory DV. Cancer's sweet tooth may be its downfall. Health and Medicine 12: 14.

16. La Vecchia C, Franceschi S, Dolara P, et al. Refined-sugar intake and the risk of colorectal cancer in humans. Int J Cancer 55 (1993): 386-389.

17. Christofk HR. The M2 splice isoform of pyruvate kinase is important for cancer metabolism and tumour growth 452 (2008): 230-233.

18. Newell K, Franchi A, Pouyssegur J, Tanock I. Studies with glycolysis-deficient cells suggest that production of lactic acid is not the only cause of tumor acidityOn the Origin of Cancer Cells (1993): 11271131.

19. Jatoi A, Ellison N, Burch PA, Sloan JA, Dakhil SR, Novotny P, Tan W, Fitch TR, Rowland KM, Young CY, Flynn PJ. A phase II trial of green tea in the treatment of patients with androgen independent metastatic prostate carcinoma. Cancer 97 (2003): 1442-1446.

20. Christofk HR, Vander Heiden MG. Wu N, Asara JM, Cantley LC. The M2 splice isoform of pyruvate kinase is important for cancer metabolism and tumour growth. Nature 452 (2008): 230-233. 
21. CW Beecher. Cancer preventive properties of varieties of Brassica oleracea. American Journal of Clinical Nutrition 59 (1994): 1166S-1170S.

22. Hutzen B, Willis W, Jones S, Cen L, Deangelis S, Fuh B, Lin JDietary agent, benzyl isothiocyanate inhibits signal transducer and activator of transcription 3 phosphorylation and collaborates with sulforaphane in the growth suppression of PANC-1 cancer cells. Cancer Cell INt 9 (2009): 24.

23. June M Chan, Peter H Gann, Edward L et al. Role of Diet in Prostate Cancer Development and Progression. Journal of Clinical Oncology 403 (2005).

24. Boehm K, Borrelli F, Ernust E, Habacher G, Hung SK, Milazzo S, Horneber Green tea (Camellia sinensis) for the prevention of cancer. Cochrane Database Syst Rev ( 2009): CD005004.

25. Jatoi A, Ellison N, Burch PA, Sloan JA, Dakhil SR, Novotny P, Ten W, Fitch TR, Rowland Km, Young CY, Flynn. A phase II trial of green tea in the treatment of patients with androgen independent metastatic prostate carcinoma. Cancer 97 (2003): 1442-1446.

26. Johnson JJ, Bailey HH, Mukhtar H. Green tea polyphenols for prostate cancer chemoprevention: a translational perspective. Green Tea May Be Useful at Preventing Prostate Cancer Phytomed (2010): 3-13.

27. Adhami MV, Mukhtar H. Anti-Oxidants from Green Tea and Pomegranate for Chemoprevention of Prostate Cancer. Molecular Biotechnology 37 (2007): 52-57.

28. Tang N, Wu Y, Zhou B, Wang B, Yu R.Green tea, black tea consumption and risk of lung cancer: a metaanalysis. Lung Cancer 65 (2009): 274-283.

29. Botelho F, Lunet N, Barros H. Coffee and gastric cancer: systematic review and meta-analysis. Cad Saude PUBLICA 22 (2006): 889-900.

30. Albert J, Tuyns, Kaaks R, Haelterman M. Colorectal cancer and the consumption of foods: A case-control study in Belgium. Nutrition and Cancer 11 (1988): 189 - 204.

31. Wargovich MJ.Nutrition and cancer: the herbal revolution. Current Opinion in Clinical Nutrition and Metabolic Care 2 (1999): 421-424.

32. Jennifer H. Cohen, Alan R. Kristal, Janet L. Stanford Fruit and Vegetable Intakes and Prostate Cancer Risk. Journal of the National Cancer Institute 92 (2000): 61-68.

33. Palmer S. Diet, nutrition, and cancer. Prog Food Nutr Sci 9 (1985): 283-341.

34. Fleischauer AT, Arab L. Garlic and cancer: A critical review of the epidemiologic literature. Journal of Nutrition 131 (2001): 1032S-1040S.

35. Gonzalez CA, Pera G, Agudo A, et al. Fruit and vegetable intake and the risk of stomach and oesophagus adenocarcinoma in the European Prospective Investigation into Cancer and Nutrition (EPIC-EURGAST). International Journal of Cancer 118 (2006): 2559-2566.

36. Ross SA, Finley JW, Milner JA. Allyl sulfur compounds from garlic modulate aberrant crypt formation. Journal of Nutrition 136 (2006): 852S-854S.

37. Gao CM, Takezaki T, Ding JH et al. Protective effect of allium vegetables against both esophageal and stomach cancer: A simultaneous case-referent study of a high-epidemic area in Jiangsu Province, China. Japanese Journal of Cancer Research 90 (1999): 614-621.

38. Milner JA. A historical perspective on garlic and cancer. J nutr 131 (2001): 1027S-1031S. 
Fortune J Health Sci 2018; 1 (1): 007-018

DOI: $10.26502 / f j h s 002$

39. Milner JA. Mechanisms by which garlic and allyl sulfur compounds suppress carcinogen bioactivation. Garlic and carcinogenesis. Adv Exp Med Biol 492 (2001): 69-81.

40. Lea MA. Organosulfur compounds and cancer.Adv Exp Med Biol 401 (1996): 147-154.

41. Rice-Evans C, Miller N, Paganga G. Free Radical Biology and Medicine 20 (2007): 933-956.

42. Trock B, Lanza E, Greenwald. Dietary fiber, vegetables, and colon cancer: critical review and metaanalyses of the epidemiologic evidence. J Natl Cancer Inst 82 (1990): 650-661.

43. Martha L Slattery, Karen P Curtin, Sandra L Edwards and Donna M Schaffer. Plant foods, fiber, and rectal cancer. American Journal of Clinical Nutrition 79 (2004): 274-281.

Citation: Muhammad Imran Qadir, Saba Irshad. Nutraceuticals for Mangement of Cancer. Fortune Journal of Health Sciences 1 (2018): 007-018.

This article is an open access article distributed under the terms and conditions of the
Creative Commons Attribution (CC-BY) license 4.0 